\title{
BMJ Open Quetiapine use in adults in the community: a population-based study in Alberta, Canada
}

\author{
Diane Duncan, ${ }^{1}$ Lara Cooke, ${ }^{1,2}$ Chris Symonds, ${ }^{1}$ David Gardner, ${ }^{3}$ \\ Tamara Pringsheim ${ }^{2,4,5}$
}

To cite: Duncan D, Cooke L, Symonds C, et al. Quetiapine use in adults in the community: a populationbased study in Alberta, Canada. BMJ Open 2016;6: e010861. doi:10.1136/ bmjopen-2015-010861

- Prepublication history for this paper is available online. To view these files please visit the journal online (http://dx.doi.org/10.1136/ bmjopen-2015-010861).

Received 17 December 2015 Revised 3 February 2016 Accepted 4 February 2016

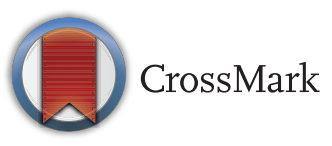

${ }^{1}$ Physician Learning Program, Continuing Medical Education and Professional Development, Cumming School of Medicine, University of Calgary, Calgary, Alberta, Canada ${ }^{2}$ Department of Clinical Neurosciences, University of Calgary, Calgary, Alberta,

Canada

${ }^{3}$ Department of Psychiatry, Dalhousie University, Halifax, Nova Scotia, Canada ${ }^{4}$ Mathison Centre for Mental Health Research and Education, University of Calgary, Calgary, Alberta, Canada

${ }^{5}$ Department of Community Health Sciences, University of Calgary, Calgary, Alberta, Canada

Correspondence to Dr Tamara Pringsheim; tmprings@ucalgary.ca

\section{ABSTRACT}

Objective: The objective of this study was to evaluate trends in prescribing of the second-generation antipsychotic medication quetiapine to adults in the province of Alberta from 2008 to 2013 through examination of dispensed prescriptions, and diagnoses associated with users of quetiapine in 2013.

Methods: We analysed administrative data from Alberta Health; the Alberta Pharmaceutical Information Network (PIN) Dispenses health data set, the Practitioner Payments (Fee-For-Service claims) health data set and the Population Registry health data set. These data sets allowed us to identify discrete quetiapine recipients for each calendar year from 2008 to 2013. To evaluate diagnoses associated with users of quetiapine, we evaluated diagnostic codes used by physicians in billings claims in 2013.

Results: Quetiapine use increased over the 6-year time period studied. In 2008, there were 16087 unique quetiapine recipients in Alberta (7.2 per 1000). By 2013, there were 35314 unique quetiapine recipients (13.3 per 1000). Use by women was higher than men at all time points. Depression was most common diagnosis associated with quetiapine recipients, which was present in $56 \%$ of users of quetiapine. Other common diagnoses associated with quetiapine use included neurotic disorders, bipolar disorder and sleep disturbances.

Conclusions: The current study of quetiapine use in the province of Alberta provides confirmatory data of the increasing use of quetiapine for the treatment of depression and anxiety disorders. Safe and rational prescribing practices must be encouraged in light of the modest advantages of quetiapine over no treatment as an adjunctive treatment of major depression, and the known harms of this medication.

\section{BACKGROUND}

Over the past decade, pharmacoepidemiological research has demonstrated increasing use of second-generation antipsychotics for the treatment of non-psychotic disorders in Canada. ${ }^{1}$ Many of these studies have evaluated specific population groups, such as

\section{Strengths and limitations of this study}

- This study used population-based provincial health administrative data to assess discrete recipients of quetiapine in the province of Alberta over a 6 -year period.

- Provincial health administrative data were used to determine diagnoses associated with quetiapine use in 2013.

- We were not able to assess the accuracy of physician selection of diagnostic codes, and there is no direct correlation between quetiapine use and diagnoses received by users.

- We were not able to assess the doses prescribed to quetiapine recipients or the duration of use.

children. ${ }^{2}{ }^{3}$ As these medications are associated with numerous, possibly serious adverse effects, including obesity and movement disorders, ${ }^{45}$ their increased use is concerning, and has prompted black box warnings from Health Canada, and drug safety monitoring initiatives. ${ }^{6}$

Two second-generation antipsychotics, quetiapine and aripiprazole, were approved by Health Canada in 2009 and 2013, respectively, as adjunctive treatments for major depressive disorders, in patients who have had incomplete responses to antidepressant medications. The marketing associated with these regulatory approvals is anticipated to further increase their use, especially considering the size of the antidepressant market. The past year prevalence of major depressive disorder in Canada is $3.9 \%$, with two-thirds seeking treatment for their symptoms. ${ }^{7}$

We performed a study in 2014 evaluating trends in antipsychotic prescriptions in Canada using the IMS Brogan databases, with a focus on the antipsychotic medication quetiapine due to our impression of increasing use of quetiapine in clinical practice. ${ }^{1}$ This study found a $300 \%$ increase in dispensed prescriptions for quetiapine ordered 
by family physicians, from 1.04 million in 2005 to 4.17 million in 2012. In comparison, dispensed prescriptions from family physicians for risperidone increased $37.4 \%$ and those for olanzapine increased $37.1 \%$. The top 4 diagnoses associated with quetiapine in 2012 were mood disorders, psychotic disorders, anxiety disorders and sleep disturbances. A 10 -fold increase in quetiapine recommendations for sleep disturbances was seen over the study period, with almost all prescribed by family physicians.

We chose to evaluate trends in the use of quetiapine by all prescribers in the province of Alberta from 2008 to 2013 to confirm and extend the results of our previous study. We used provincial administrative data and focused on adults aged 19-64 years for our analysis as many previous pharmacoepidemiological studies of antipsychotic use in Canada have focused on children or the elderly. Canada has a public national healthcare service. Coverage for prescription medications varies by province. In the province of Alberta, prescriptions are paid out-of-pocket or by third party payers except for individuals receiving social assistance or those over 65 years of age. We hypothesised that quetiapine prescribing in Alberta would increase over this time period, and that most prescriptions would be for the treatment of non-psychotic disorders.

\section{METHODS}

\section{Ethics}

Ethics approval was received from the University of Calgary Conjoint Health Research Ethics Board (REB13-0459) to use aggregate data on medication use linked to diagnoses as a means of educating and informing physicians about how data with feedback can be used as an effective, innovative Continuing Professional Development (CPD) activity.

\section{Data sources}

The Alberta Pharmaceutical Information Network (PIN) Dispenses health data set is an Alberta Health administrative database that captures information on medications dispensed by community pharmacies; about $95 \%$ of community pharmacies submit records to PIN. ${ }^{8}$ The PIN data set includes information on each dispense event and associated information on patient, prescriber, dispenser, facility and drug. ${ }^{8}$

The Practitioner Payments (Fee-For-Service (FFS) claims) health data set is an Alberta Health administrative database that captures information on FFS claims for insured health services. The FFS claims data set includes recipient, provider and service data. ${ }^{8}$

The Population Registry health data set is an Alberta Health administrative database that captures basic demographic and geographic information of Albertans who are registered with the Alberta Health Care Insurance Plan (AHCIP). ${ }^{8}$ All residents of Alberta are eligible for coverage by the AHCIP, with the exception of those who have active health coverage in another province, and members of the Canadian Forces. Albertans must be registered with the plan in order to receive insured hospital and physician services. As there is no private healthcare system in Alberta, this is the only way to receive healthcare services. Therefore, nearly $100 \%$ of the Alberta population is covered by the AHCIP, and Alberta Health uses the AHCIP registry to estimate the population of Alberta. The rates of use of quetiapine being presented in our study are therefore proportions of the total population of Alberta. The population estimates from the AHCIP registry are comparable to Statistics Canada estimates, with differences less than $5 \%$ on yearly estimates.

Administrative data from the PIN data set were used to identify quetiapine recipients, aged 19-64 years, from 2008 to 2013. Population data from the AHCIP registry files were used to calculate rate of quetiapine recipients. Administrative data from the FFS claims data set were used to identify diagnoses of interest for quetiapine recipients in 2013.

Drug products in the PIN data set are identified by unique Drug Identification Number (DIN). A list of DINs for quetiapine was created from the Health Canada Drug Product Database (DPD) data extract files; 89 DINs for active products and 1 DIN for inactive products were identified. ${ }^{9}$ PIN dispensing records for quetiapine from 1 January 2008 to 31 December 2013 were identified using the DIN list. The dispensing records were analysed to identify discrete quetiapine recipients, aged 19-64 years, for each calendar year. Quetiapine recipients were only counted once in a calendar year. Quetiapine recipients with dispensing records for more than one calendar year were counted as a recipient for each year in which they had one of more dispensing records for quetiapine. Quetiapine dose and days supply was not captured. The individual's date of birth in the PIN data set was used to determine age assignment, as of 31 December for each calendar year. The individual's Unique Lifetime Identifier (ULI) in the PIN data set and 'count distinct' function in SAS were used to identify discrete recipients. Recipients were removed if the PIN data set did not include their date of birth or postal code; recipients were also removed if their postal code in the PIN data set was located outside Alberta. The sex of discrete recipients as recorded in the PIN data set was identified for descriptive analysis. All rate denominators were calculated using estimates from the AHCIP population registry files, stratified by year and age. Rates are reported as number of discrete recipients per 1000 population. All physician prescriber specialties were included.

Physicians are required to enter a diagnosis for every billed service using Alberta Health Diagnostic Codes (International Classification of Diseases, Ninth Revision, Clinical Modification, ICD-9-CM); physicians can enter up to three diagnoses per claim. ${ }^{10}$ Diagnoses in the FFS claims data set were matched to the discrete quetiapine 
Table 1 Diagnostic codes of interest

\begin{tabular}{ll}
\hline Diagnoses & $\begin{array}{l}\text { Diagnostic codes } \\
\text { (ICD-9-CM) }\end{array}$ \\
\hline Dementia & $290 . x$ \\
Delirium & $293 . x$ \\
Other organic psychotic & $294 . x$ \\
conditions & \\
Schizophrenia & $295 . x, 297 . x$ \\
Bipolar disorder & $296 . x$ \\
Other non-organic psychoses & $298 . x$ \\
Psychoses origin specific to & $299 . x$ \\
childhood & \\
Neurotic disorders & $300 . x$ (excluding 300.4) \\
Neurotic depression & 300.4 \\
Drug dependence & $304 . x$ \\
Adjustment reaction & $309 . x$ \\
Depression & 311 \\
Conduct disorder & $312 . x$ and 313.x \\
ADHD & $314 . x$ \\
Alzheimer's & $331 . x$ \\
Sleep disturbances & 780.5 \\
Nervousness & 799.2 \\
\hline ICD-9-CM; International Classification of Diseases, Ninth \\
Revision, Clinical Modification. & \\
&
\end{tabular}

recipients identified from the PIN data set using ULI for the calendar year 2013; quetiapine recipients with no billing data in 2013 were excluded from subsequent analysis. In order to be considered in the analysis, individuals were required to have one or more quetiapine dispense records in the calendar year 2013 in the PIN data set AND one or more billed services in the FFS claims data set in the calendar year 2013. ICD-9 codes of interest were identified by the principal investigator. All diagnostic codes of interest billed by any practitioner, at any time in the calendar year 2013, were identified. Billed diagnostic code of interest were not correlated with quetiapine dispense event. Diagnostic codes (ICD-9-CM) of interest were grouped into diagnoses based on consultation with the principal investigator and coding experts (see table 1). Analysis for diagnoses of interest was performed; primary, secondary and tertiary diagnoses were analysed.

\section{RESULTS}

In 2008, there were 16087 unique quetiapine recipients, aged 19-64 years, in Alberta; with 9650 female recipients and 6437 male recipients. By 2013, there were 35314 unique quetiapine recipients, with 20577 female recipients and 14737 male recipients. In 2008, the rate of quetiapine recipients was 7.2 (8.6 females; 5.7 males) per 1000 population. In 2013, the rate of quetiapine recipients was 13.3 (15.8 females; 10.9 males) per 1000 population, representing a 1.85-fold increase within 5 years (see figure 1 ).

For the calendar year 2013, diagnoses in the FFS claims data set were matched to quetiapine recipients; 95\% (33 496) of recipients had billing data for any service. The $5 \%$ (1818) of quetiapine recipients with no billing data were excluded from subsequent analysis.

In 2013, recipients of quetiapine with billing data had 0-10 of the diagnoses of interest specified in table 1; most recipients had one or two diagnoses of interest (see table 2). Depression, neurotic disorders and bipolar disorder were the most common diagnoses of interest associated with recipients of quetiapine (table 3 ); $56 \%$ (18 717) of this cohort of quetiapine recipients had a diagnosis of depression. In total, $35.5 \%$ (11 899) of quetiapine recipients had only one diagnosis of interest; depression $(38.4 \%)$, neurotic disorders $(24.3 \%)$ and bipolar disorder $(14.5 \%)$ were the most common single diagnosis of interest (see table 4 ).

\section{DISCUSSION}

Our analysis of quetiapine use using Alberta provincial databases has confirmed and extended the results of our previous national Canadian study. One of the key advantages of the current provincial study over our previous analysis of national data is the ability to look at the
Figure 1 Rate of recipients, 1964 years, of quetipine by sex, 2008-2013 (Alberta).




Table 2 Number of diagnostic codes of interest for quetiapine recipients in 2013

\begin{tabular}{lc}
\hline Number of diagnoses & Number of recipients \\
\hline 0 & 3129 \\
1 & 11899 \\
2 & 9321 \\
3 & 5007 \\
4 & 2331 \\
5 & 1091 \\
6 & 455 \\
7 & 172 \\
8 & 56 \\
9 & 32 \\
10 & 3 \\
Total recipients & 33496 \\
\hline Diagnoses of interest for quetiapine recipients, $19-64$ years, in \\
2013 (Alberta).
\end{tabular}

number of discrete individuals receiving quetiapine in the province of Alberta. Our previous analysis only allowed us to evaluate the number of dispensed prescriptions, and as a single individual may fill several prescriptions for the same medication over a period of 1 year, we were unable to determine the number of individuals receiving the dispensed prescriptions. The number of quetiapine recipients in Alberta has nearly doubled over 6 years, with 16 per 1000 women and 11 per 1000 men aged 19-64 receiving quetiapine in 2013.

The current study uses a different method for evaluating diagnoses associated with quetiapine use than our previous study, with the two studies therefore providing

Table 3 Diagnostic codes associated with quetiapine recipients in 2013

\begin{tabular}{lc}
\hline Diagnoses of interest & $\begin{array}{c}\text { Number of } \\
\text { recipients }\end{array}$ \\
\hline Depression & 18717 \\
Neurotic disorders & 16109 \\
Bipolar disorder & 9790 \\
Sleep disturbances & 4096 \\
Adjustment reaction & 3278 \\
Drug dependence & 3276 \\
None & 3129 \\
Schizophrenia & 2995 \\
Neurotic depression & 1899 \\
Other non-organic psychoses & 1697 \\
Conduct disorder & 1057 \\
ADHD & 748 \\
Dementia & 410 \\
Delirium & 372 \\
Other organic psychotic conditions & 344 \\
Psychoses origin specific to childhood & 153 \\
Alzheimer's & 91 \\
Nervousness & 9 \\
\hline Quetiapine recipients, 19-64 years, and diagnoses of interest in \\
2013 (Alberta). \\
In total, 33 496 recipients had 0-10 diagnoses of interest. \\
\hline
\end{tabular}

complementary information. Our previous national study provided information on diagnoses associated with quetiapine prescriptions through a physician panel study, in which a sample of Canadian office-based physicians recorded the therapeutic indication for each drug recommended for a 2-day period four times per year. The current study used data on diagnoses obtained through physician billing claims. Recipients of quetiapine in 2013 had 0-10 diagnoses of interest. Depression was the most common diagnosis associated with quetiapine use, present in $56 \%$ of users. A greater proportion of individuals receiving quetiapine had a diagnosis of neurotic disorder (48\%) or sleep disturbances (12\%) than schizophrenia $(9 \%)$ or other non-organic psychoses $(5 \%)$, highlighting the fact that the vast majority of quetiapine recipients do not have psychotic disorders, and that off-label use of quetiapine is frequent.

One likely contributing factor to the increased prescribing of quetiapine is the extension of its indications. In 2009, Health Canada approved quetiapine for the adjunctive treatment of major depression not responding adequately to antidepressants. However, we cannot determine from our data if quetiapine use is limited to this subgroup of patients with depression or a larger group nor the clinical outcomes associated with its use in general practice. The second most common diagnosis of interest associated with quetiapine recipients was neurotic disorders, a billing code that identifies people with various anxiety disorders. The ICD9 codes for neurotic disorders (300) includes anxiety disorders, hysteria, phobic state, obsessive compulsive disorder, neurasthenia, depersonalisation syndrome and hypochondriasis. While some of these terms are now outdated, they correspond to Diagnostic and Statistical Manual of Mental Disorders, Fifth Edition (DSM V) diagnoses of anxiety disorders, obsessive compulsive and related disorders, dissociative disorders, and somatic symptom and related disorders. Quetiapine is not approved by any regulatory body internationally for the treatment of any anxiety disorder. As quetiapine use expands extensively into the population, with the rate of off-label use approaching its approved uses, the issue of informed decision-making is highly relevant. It is important that clinicians inform patients of their treatment options and of the potential benefits and risks of each, particularly when used off-label.

When treating adults with depression not sufficiently responsive to antidepressants with adjunctive quetiapine, the number needed to treat for remission is $9(95 \%$ CI 6 to 19) compared with no treatment (ie, placebo) over $6-8$ weeks. The number needed to treat for adverse effects-related discontinuation is 8 (95\% CI 6 to 11), and the number needed to harm for adverse metabolic laboratory results is 6 (95\% CI 4 to 9$).{ }^{11}$ The rates of clinically significant weight gain in these short-term trials were $7 \%$ for quetiapine $300 \mathrm{mg} /$ day vs $2 \%$ with placebo. Data on long-term adverse effects, including rates of obesity, dyslipidaemia, diabetes mellitus and 
Table 4 Diagnostic codes associated with quetiapine recipients with one diagnosis of interest in 2013

\begin{tabular}{|c|c|c|c|c|c|c|}
\hline Diagnosis & Female & Per cent & Male & Per cent & Total & Per cent \\
\hline Depression & 2943 & 42.15 & 1625 & 33.05 & 4568 & 38.38 \\
\hline Neurotic disorders & 1731 & 24.79 & 1164 & 23.67 & 2895 & 24.33 \\
\hline Bipolar disorder & 997 & 14.28 & 724 & 14.72 & 1721 & 14.46 \\
\hline Sleep disturbances & 413 & 5.92 & 372 & 7.57 & 785 & 6.60 \\
\hline Schizophrenia & 208 & 2.98 & 372 & 7.57 & 580 & 4.87 \\
\hline Drug dependence & 153 & 2.19 & 205 & 4.17 & 358 & 3.01 \\
\hline Adjustment reaction & 184 & 2.64 & 139 & 2.83 & 323 & 2.71 \\
\hline Neurotic depression & 153 & 2.19 & 88 & 1.79 & 241 & 2.03 \\
\hline Conduct disorder & 44 & 0.63 & 67 & 1.36 & 111 & 0.93 \\
\hline ADHD & 34 & 0.49 & 47 & 0.96 & 81 & 0.68 \\
\hline Psychoses origin specific to childhood & 13 & 0.19 & 35 & 0.71 & 48 & 0.40 \\
\hline Other non-organic psychoses & 64 & 0.92 & 32 & 0.65 & 96 & 0.81 \\
\hline Dementia & 23 & 0.33 & 16 & 0.33 & 39 & 0.33 \\
\hline Delirium & 12 & 0.17 & 13 & 0.26 & 25 & 0.21 \\
\hline Other organic psychotic conditions & 8 & 0.11 & 13 & 0.26 & 21 & 0.18 \\
\hline Alzheimer's & 2 & 0.03 & 5 & 0.10 & 7 & 0.06 \\
\hline Total & 6982 & 100 & 4917 & 100 & 11899 & 100 \\
\hline
\end{tabular}

movement disorders, when used as an adjunctive treatment for depression are scarce.

The use of quetiapine for the treatment of anxiety disorders has been evaluated in several trials versus placebo and SSRIs. ${ }^{12}$ Quetiapine reduced anxiety more than placebo, by 2.6 points (95\% CI 1.2 to 4.0 ) on the Hamilton Anxiety Rating Scale. The number needed to treat for remission was 10 , while the number needed for discontinuation due to adverse effects was 8 . Trials comparing quetiapine to paroxetine and escitalopram have found similar efficacy between agents but poorer tolerability with quetiapine. The manufacturer of quetiapine withdrew its applications for anxiety disorders indication, presumably due to concerns raised by the Food and Drug Administration (FDA) about safety and tolerability. ${ }^{13}$

Given the potential for harm associated with quetiapine use and the increasing use of this medication in adults, safety monitoring is essential. Individuals prescribed quetiapine for any reason should have baseline measurement of body mass index; waist circumference; blood pressure; extrapyramidal symptoms; and laboratory tests of cholesterol, triglycerides, glucose, liver enzymes and thyroid-stimulating hormone. Follow-up clinical assessment should take place at 2 weeks, 1, 3, 6 and 12 months; follow-up laboratory tests are recommended at 3 and 12 months. ${ }^{5}$

Limitations of the current study include that individuals who are not eligible for AHCIP coverage are not included in the Practitioner Payments (FFS claims) administrative data set. Individuals who are not eligible for AHCIP include those with active health coverage in another province, those who have formally opted out of the AHCIP, those not yet registered with Alberta Health, those with an inactive AHCIP, Federal Penitentiary Inmates and Canadian Forces personnel. ${ }^{14}$ Practitioners may not select the most accurate diagnostic code when billing for services; practitioners may select commonly used diagnostic codes when submitting claims for service rather than the most accurate diagnostic code. A recent analysis of Alberta-wide physician claims data to examine the face validity of clinical information found that nearly half of ICD-9 diagnostic codes used were more detailed than required (four-digit or five-digit coding rather than three-digit minimum), and that the most frequently submitted reason for visit was consistent with the physician specialty. ${ }^{15}$ In this study, we were not able to assess the accuracy of physician selection of diagnostic codes used, and there is no direct correlation between quetiapine prescription and diagnoses. The practitioner who prescribed quetiapine may be different than the practitioner who submitted a claim with a diagnosis of interest for the recipient of quetiapine.

Our data pertain to individuals residing in the province of Alberta, which provides universal healthcare access and coverage for prescription medications for disadvantaged people. Our results are in keeping with our previous study evaluating national prescribing trends, as well as pharmacoepidemiological studies from other developed countries. Ilyas and Moncrieff found an increase in quetiapine prescription items in England over time, from approximately 200000 prescription items in 2002, to over 1600000 prescription items in 2010. In 2010, $54 \%$ of items were for $25 \mathrm{mg}$ tablets. ${ }^{16} \mathrm{~A}$ study of antipsychotic prescribing in UK primary care between 2007 and 2011 found that the most common diagnoses associated with quetiapine use were depression, dementia, anxiety and sleep disorders. ${ }^{17}$ A US study looking at diagnoses associated with atypical antipsychotics in a claims database found that major depressive disorders and bipolar disorder were the most common diagnoses associated with quetiapine use. 
Of those prescribed quetiapine for a non-approved indication, anxiety disorders and substance abuse were the most common diagnoses. ${ }^{18}$ We were not able to evaluate the dose of quetiapine or duration of quetiapine use by individual recipients. We therefore are unable to comment on the most frequently prescribed dosages of quetiapine or whether use is short term or long term in the majority of users. Such information would be of value in understanding physician prescribing practices for quetiapine for non-psychotic disorders, as well as better understanding the risk of harm.

The current study of quetiapine use in the province of Alberta provides confirmatory data of the increasing use of quetiapine for the treatment of depression and anxiety disorders. Safe and rational prescribing practices must be encouraged in light of the modest advantages of quetiapine over no treatment as an adjunctive treatment of major depression, and the known harms of this medication. Alternative adjunctive treatments for major depression should be considered by prescribers and reviewed with their patients, with preference given to those treatments associated with the least harm. Further research should focus on the most commonly prescribed doses of quetiapine, and adverse effects associated with long-term use.

Acknowledgements The authors acknowledge Mr Neil Gaudette from Alberta Health for his assistance accessing the Alberta Health data sets. This study is based in part on data provided by Alberta Health.

Contributors DD helped design the study, directed the data analysis, wrote the Methods and Results sections of the manuscript, and critically reviewed and revised the rest of the manuscript. LC and CS helped design the study, and critically reviewed and revised the manuscript. DG critically reviewed and revised the manuscript. TP helped design the study, wrote the Background and Discussion sections of the manuscript, and critically reviewed and revised the rest of the manuscript.

Funding This research received no specific grant from any funding agency in the public, commercial or not-for-profit sectors.

Disclaimer The interpretation and conclusions contained here are those of the researchers and do not necessarily represent the views of the Government of Alberta. Neither the Government of Alberta nor Alberta Health expresses any opinion in relation to this study.

Competing interests None declared.

Ethics approval Ethics approval was received from the University of Calgary Conjoint Health Research Ethics Board (REB13-0459).

Provenance and peer review Not commissioned; externally peer reviewed.
Data sharing statement No additional data are available.

Open Access This is an Open Access article distributed in accordance with the Creative Commons Attribution Non Commercial (CC BY-NC 4.0) license, which permits others to distribute, remix, adapt, build upon this work noncommercially, and license their derivative works on different terms, provided the original work is properly cited and the use is non-commercial. See: http:// creativecommons.org/licenses/by-nc/4.0/

\section{REFERENCES}

1. Pringsheim T, Gardner DM. Dispensed prescriptions for quetiapine and other second generation antipsychotics in Canada from 2005 to 2012: a descriptive study. CMAJ Open 2014;2:E225-32.

2. Pringsheim T, Lam D, Patten SB. The pharmacoepidemiology of antipsychotic medications for Canadian children and adolescents, 2005-2009. J Child Adolesc Psychopharmacol 2011;21:1-8.

3. Alessi-Severini S, Biscontri RG, Collins DM, et al. Ten years of antipsychotic prescribing to children: a Canadian population-based study. Can J Psychiatry 2012;57:52-8.

4. Pringsheim T, Lam D, Ching $\mathrm{H}$, et al. Metabolic and neurological complications of second generation antipsychotic use in children. Drug Saf 2011;34:651-68.

5. Gardner DM, Teehan MD. Antipsychotics and their side effects. Cambridge: Cambridge University Press, 2010.

6. Pringsheim T, Panagiotopoulos C, Davidson J, et al. Evidence based recommendations for monitoring safety of second generation antipsychotics in children and youth. Pediatr Child Health 2011;16:581-9.

7. Patten SB, Williams JVA, Lavorato $\mathrm{DH}$, et al. Descriptive epidemiology of major depressive disorder in Canada in 2012. Can J Psychiatry 2015;60:23-30.

8. Overview of Administrative Health Datasets2015 2015.01.13. http:// www.health.alberta.ca/documents/Research-Health-Datasets.pdf

9. Health Canada Drug Product Database Data Extract Files2015 2015.01.13. http://hc-sc.gc.ca/dhp-mps/prodpharma/databasdon/ dpd_bdpp_data_extract-eng.php

10. Alberta Health Claims Assessment Alberta Health Diagnostic Codes2015 2015.01.13. http://www.health.alberta.ca/documents/ Diagnostic-Code-ICD-9.pdf

11. Pringsheim T, Gardner D, Patten SB. Adjunctive treatment with quetiapine for major depressive disorder: are the benefits of treatment worth the risks? BMJ 2015;350:h569.

12. Depping AM, Komossa K, Kissling W, et al. Second generation antipsychotics for anxiety disorders. Cochrane Database Syst Rev 2010;(12):CD008120.

13. Kuehn B. FDA panel issues mixed decision on quetiapine in depression and anxiety. JAMA 2009;301:2081-2.

14. Allied Health Practitioner's Resource Guide2015 2015.01.13. http:// www.health.alberta.ca/documents/Allied-Practitioners-Guide-2014. pdf

15. Cunningham CT, Cai $P$, Topps $D$, et al. Mining rich health data from Canadian physician claims: features and face validity. BMC Res Notes 2014;7:682.

16. Ilyas S, Moncrieff $\mathrm{J}$. Trends in prescriptions and costs of drugs for mental disorders in England, 1998-2010. Br J Psychiatry 2012;200:393-8.

17. Marston L, Nazareth I, Petersen I, et al. Prescribing antipsychotics in UK primary care: a cohort study. BMJ Open 2014;4:e006135.

18. Citrome L, Kalsekar I, Guo Z, et al. Diagnoses associated with use of atypical antipsychotic in a commercial health plan: a claims database analysis. Clin Ther 2013;35:1867-75. 